\title{
Transport Analysis and Model for the Performance of an Ultrasonically Enhanced Filtration Process
}

\author{
Michael T. Grossner \\ Case Western Reserve University \\ Joanne M. Belovich \\ Equlvelathissanteafhlitiershty works at: https://engagedscholarship.csuohio.edu/encbe_facpub \\ Donart of the Meke \\ dase doesterfireserte thisuefsity benefit you? Let us know! \\ Publisher's Statement
}

NOTICE: this is the author's version of a work that was accepted for publication in Chemical

Engineering Science. Changes resulting from the publishing process, such as peer review, editing, corrections, structural formatting, and other quality control mechanisms may not be reflected in this document. Changes may have been made to this work since it was submitted for publication. A definitive version was subsequently published in Chemical Engineering Science, 60, 12, (June 2005) DOI 10.1016/j.ces.2005.01.005

\section{Original Citation}

Grossner, M. T., Belovich, J. M., , \& Feke, D. L. (2005). Transport analysis and model for the performance of an ultrasonically enhanced filtration process. Chemical Engineering Science, 60(12), 3233-3238.

doi:10.1016/j.ces.2005.01.005

\section{Repository Citation}

Grossner, Michael T.; Belovich, Joanne M.; and Feke, Donald L., "Transport Analysis and Model for the Performance of an Ultrasonically Enhanced Filtration Process" (2005). Chemical \& Biomedical Engineering Faculty Publications. 45.

https://engagedscholarship.csuohio.edu/encbe_facpub/45

This Article is brought to you for free and open access by the Chemical \& Biomedical Engineering Department at EngagedScholarship@CSU. It has been accepted for inclusion in Chemical \& Biomedical Engineering Faculty Publications by an authorized administrator of EngagedScholarship@CSU. For more information, please contact library.es@csuohio.edu. 


\title{
Transport analysis and model for the performance of an ultrasonically enhanced filtration process
}

\author{
Michael T. Grossner ${ }^{\mathrm{a}}$, Joanne M. Belovich ${ }^{\mathrm{b}}$, Donald L. Feke ${ }^{\mathrm{a}, *}$ \\ ${ }^{a}$ Department of Chemical Engineering, Case Western Reserve University, 10900 Euclid Avenue, Cleveland, OH 44106, USA \\ ${ }^{\mathrm{b}}$ Department of Chemical and Biomedical Engineering. Cleveland State University, 2121 Euclid Avenue, Cleveland, OH 44115, USA
}

\section{Introduction}

A process for the filtration of small suspended particles using a high-porosity polyester mesh situated in a resonant ultrasonic field has been reported recently (Gupta and Feke, 1997, 1998; Wang et al., 2004). A schematic of this filtration concept is depicted in Fig. 1. Shown is a rectangular chamber in which the polymer mesh is sandwiched between a piezoelectric transducer and a glass reflector. As suspension flows through the mesh when the sound field is active, small particles are entrapped even though the pores of the mesh are approximately two orders of magnitude larger than the particle diameter. Upon deactivation of the acoustic field, the particles are released from the mesh.

The basis for the particle entrapment arises from complex interactions between the mesh, the acoustic and hydrodynamic flow fields, and the particles themselves. The acoustic field, scattered from the mesh elements, results in acoustic forces that attract particles toward the collector. The relevant transport phenomena active on the length scale of the particles been modeled and experimentally verified. This microscale analysis focuses on the motion of individual particles in the vicinity of one collection element comprising the mesh (Grossner et al., 2003, 2005). While this single-collector model is an excellent tool to understand the underlying fundamental phenomena active in the acoustic filtration process, it alone is not sufficient to predict the macroscopic performance of such a filter system. Here, we seek to develop a model that combines information from the single-collector studies and properties of the overall filtration system that leads to predictions of important process characteristics such as particle breakthrough times and the general retention performance of the acoustic separator.

An approach taken by investigators of high gradient magnetic separation (HGMS) provides the starting point for this analysis (Gerber and Birss, 1983). In HGMS, a steel mesh (similar to steel wool) is magnetized to collect small 


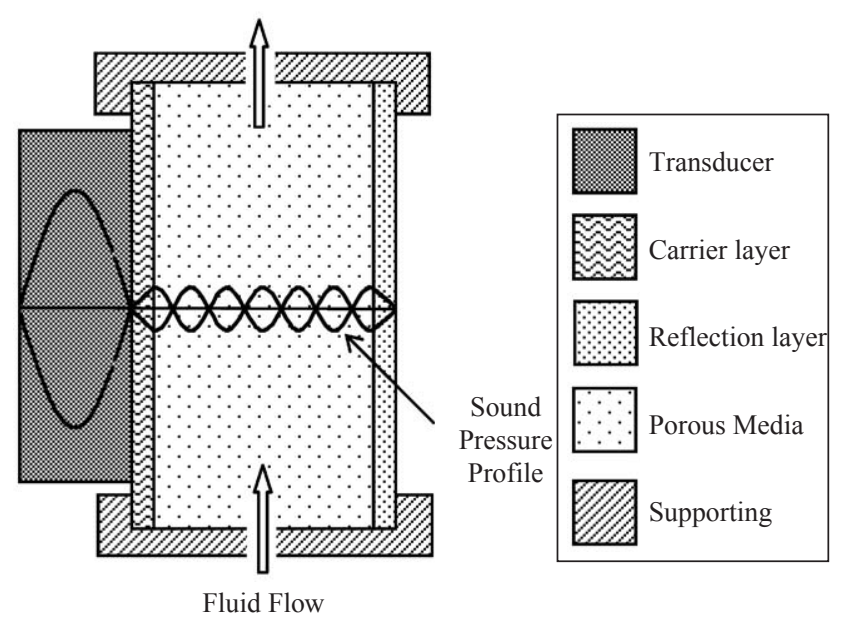

Fig. 1. A schematic of the acoustic filtration process.

magneticallysusceptible particles from a suspension. This process has been examined microscopically( Gerber and Birss, 1983) in a similar manner as the acoustic separation process in this research. Once a capture radius has been defined for a single collector (akin to the "capture window" discussed in a previous paper (Grossner et al., 2003)), a multi-collector model is assembled to form a model of the entire separator (Gerber and Watmough, 1982).

In this paper, we extend this approach to derive a performance model for the acoustic filtration process. Numerical simulations of the model provide predictions of the spatial and temporal evolution of the concentration of particles captured within the mesh. These model results are further compared to the results of experiments in which macroscopic performance parameters, such as particle breakthrough times, are reported.

\section{Derivation of the transport model}

\subsection{Coordinate system and important parameters}

The overall operation and performance of the filtration device is modeled on the basis of a conservation relation for the suspended particles. Consistent with the configuration within the experimental trials, the model assumes dependence on onlyone spatial variable. A schematic with coordinate system definitions are presented in Fig. 2. The chamber has length $L$ in the flow direction and a cross-sectional area of $S$. The independent variable in the flow direction is $x$. Due to the presence of the mesh, the convective flow is taken to be a one-dimensional plug flow with superficial velocity $v_{0}$.

Two variables are used to describe the spatial and temporal distribution particles within the chamber. First, $C(x, t)$ is used to denote the concentration of particles in free suspension (not trapped within the mesh), and has units of the number of particles per volume of fluid. The variable $N(x, t)$ denotes the particle retention density, or number of trapped

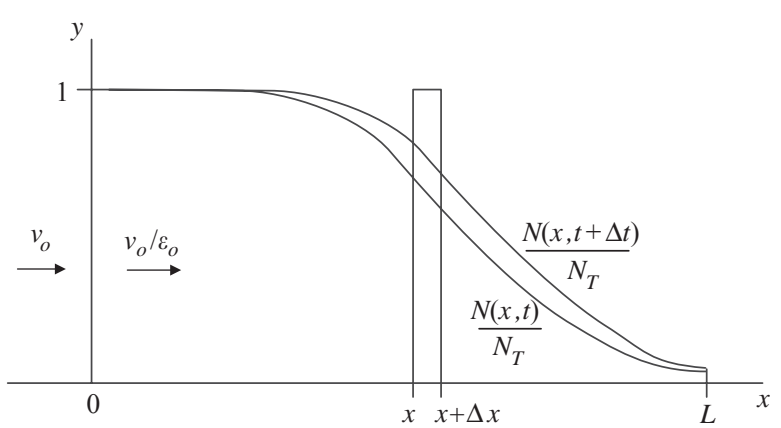

Fig. 2. Schematic depicting the coordinate system used for the model. The suspension flows (superficial velocity $v_{0}$ ) into the mesh (porosity $\varepsilon_{0}$ ) at $x=0$ and exits at $x=L ; N_{T}$ is the concentration of captured particles at saturation. The temporal variable is $t$.

particles per unit volume of the mesh, and $N_{T}$ is used to indicate the saturation value of $N$ inside a particular mesh. With the concentration of the entering suspension fed to the mesh $C(0, t)$ specified as a boundarycondition and the initial loading of the mesh $N(x, 0)$ specified as an initial condition, the model intends to predict how $C$ and $N$ change with position and time during operation of the filtration process.

\subsection{Particle conservation model}

A material balance for a differential section of the mesh is used as the basis for a model relating $C$ and $N$ to position and time. Since the particles are presumed to be relativelylar ge (tens of microns), diffusion is neglected. We also assume that the mesh has a uniform porosity $\varepsilon_{0}$ throughout. As particles become trapped, the free volume within the mesh decreases. However, in the experimental trials, $\varepsilon_{0}$ is high (typically $95 \mathrm{vol} \%$ ) and solids loading within the mesh is typically below $10 \mathrm{vol} \%$. Thus, we use the single parameter $\varepsilon_{0}$ to approximate the mesh porositythroughout the course of the particle collection process. A material balance on particles with the mesh is then

$$
\frac{\partial}{\partial t}\left(N+\varepsilon_{0} C\right)+v_{0} \frac{\partial C}{\partial x}=0 .
$$

To close this model, an expression relating the conversion of free to trapped particles (the functional relationship between $N$ and $C$ ) must be developed. Our previous microscale analysis of the efficiency of individual collectors to capture suspended particles provides the basis for this relationship. Here, we extend the results of the single-collector analysis to apply to the multiple collectors present within the actual mesh.

The abilityof an individual collection element to capture particles can be quantified in terms of a capture window (Grossner et al., 2003). This is the area, upstream from the collectors, defined such that particles that flow through this window eventuallybecome associated with the collector element. Particles that do not flow though the upstream capture window will flow around the collector. The single-collector 
model assumes that the cylindrical collector (uniform radius $R_{c}$, average length $L_{c}$ ) is aligned perpendicular to the flow and to the acoustic field. Based on a trajectoryanaly sis, the dimensions of the upstream capture window (area $A_{\text {cap }}$ ) are determined as a function of acoustic and flow conditions. In general, the capture area is larger than the actual area projected bythe collector when an appropriate acoustic field is applied. For convenience, we define a dimensionless capture radius as

$R_{\text {cap }}^{*} \equiv \frac{A_{\text {cap }}}{2 R_{c} L_{c}}$.

For favorable acoustic conditions, $R_{\text {cap }}^{*}>1$.

In order to extend the single-collector results to the configuration of a random mesh, we adopt a conceptual model for the mesh structure. The number of individual collectors per volume within the mesh maybe estimated from its porosity as

$n_{c}=\frac{\text { fraction of solids comprising the mesh }}{\text { average volume of one collector }}=\frac{1-\varepsilon_{0}}{\pi R_{c}^{2} L_{c}}$.

Within a differential volume of the mesh (cross-sectional area $S$ and length $\Delta x$ ), the total number of collectors is $n_{c} S \Delta x$. However, since the collectors are oriented randomly to the flow and acoustic field, the total capture area presented bythese collectors is not simplythe product of the number of collectors and the value of $A_{\text {cap }}$ derived from the single-collector analysis. We thus introduce a collector orientation parameter $\langle f\rangle$ such that the total effective capture area projected perpendicular to the superficial flow for the differential section of the mesh is

$A_{\text {cap }, \text { tot }}=\langle f\rangle A_{\text {cap }} n_{c} S \Delta x$

and the probability, $p$, of capturing an individual particle flowing through the differential volume is given by

$p=A_{\text {cap }, \text { tot }} / S=\langle f\rangle A_{\text {cap }} n_{c} \Delta x$.

The rate of particle trapping in the differential element is given bythe rate at which particles flow through the differential volume times the probabilityof their capture. Thus

$\frac{\partial(N S \Delta x)}{\partial t}=S v_{0} p C$

which upon substitution of Eqs. (2)-(5) and subsequent simplification yields

$\frac{\partial N}{\partial t}=v_{0}\langle f\rangle \frac{2\left(1-\varepsilon_{0}\right) R_{\text {cap }}^{*}}{\pi R_{C}} C$.

Finally, we recognize that the capture radius is expected to diminish as the number of previouslycaptured particles increases. Thus, we follow the lead of Gerber and Birss (1983) and introduce a correction of the form

$R_{\text {cap }}^{*}=R_{\text {cap }}^{0 *}\left(1-\frac{N}{N_{T}}\right)^{\gamma}$, where $R_{\text {cap }}^{0 *}$ represents the dimensionless capture radius for a clean collector. The empirical exponent $\gamma$ depends on the general operating conditions, but is typically between 0.1 and 2 . With the definition

$L_{0} \equiv \frac{\pi R_{c}}{2\left(1-\varepsilon_{0}\right)\langle f\rangle R_{\text {cap }}^{0 *}}$.

Eq. (7) becomes

$\frac{\partial N}{\partial t}=\frac{v_{0}}{L_{0}}\left(1-\frac{N}{N_{T}}\right)^{\gamma} C$.

\section{Solution method}

Eqs. (1) and (10) form a coupled system of equations that give $N(t, x)$ and $C(t, x)$. For convenience, we rewrite these equations in terms of a new set of independent variables $(\tau, X)$ where

$\tau=t-\varepsilon_{0} x / v_{0}$

is the displacement time, and

$X=x$

is the new spatial coordinate. Using these variables, Eq. (1) can be transformed into

$\frac{\partial N}{\partial \tau}+v_{0} \frac{\partial C}{\partial X}=0$

while Eq. (10) becomes

$\frac{\partial N}{\partial \tau}=\frac{v_{0}}{L_{0}}\left(1-\frac{N}{N_{T}}\right)^{\gamma} C$.

Eqs. (13) and (14) form a coupled system of first-order equations in $(\tau, X)$ space.

In the experimental trials, filtration is typically performed under the condition

$C(\tau, X=0)=C_{0}$

which reflects a suspension of constant composition fed at the entrance to the mesh. Two different categories of experiments have been performed. In one type, the acoustic field is applied prior to the introduction of particles to the mesh. To simulate this type of experiment, we use

$N(\tau=0, X)=0$.

Imbedded in this condition is the constraint that no particles can be trapped in the mesh at position $x$ earlier than the flow transport time to that position $\left(x / v_{0}\right)$.

For this case, the model equations were solved using a finite difference method suggested by Gerber and Watmough (1982). First, Eq. (14) is solved for $x=0$ using the Runge-Kutta method to give $N(0, \tau)$. From this result, $\partial N / \partial \tau$ is computed and substituted back into Eq. (13) to 
obtain $\partial C / \partial x$. Then, the free particle concentration at the next $x$ position is calculated from

$C(0+x, \tau)=C(0, \tau)+\frac{\partial C}{\partial x} \quad x$.

The process of alternatelysolving Eq. (14) then Eq. (13) is then repeated one spatial layer at a time until the end of the separator $x=L$ is reached.

In the second type of experiment, suspension flow through the mesh is established prior to the application of the acoustic field and thus particles will be present at all positions within the mesh when the acoustic field is applied. Thus, since $C(t=0, x)=C_{0}$ and $N(t=0, x)=0$, the boundaryconditions for this case become

$C\left(\tau=-\varepsilon_{0} x / v_{0}, X\right)=C_{0}$,

$N\left(\tau=-\varepsilon_{0} x / v_{0}, X\right)=0$.

The same solution algorithm as described above can be applied to this case as well. However, the solution grid must be designed such that

$$
\tau=\varepsilon_{0} \quad x / v_{0}
$$

and the solution domain starts along the line $\tau=-\varepsilon_{0} x / v_{0}$.

\section{Results and discussion}

The filtration performance model described above has been applied to simulate the two types of experiments that demonstrate the ultrasonicallyassisted mesh-filtration process. For our simulations, we selected the value of $\gamma=2$, based on trial runs of the model using values of $\gamma$ between 0.1 and 2. This value of the deterioration parameter was not adjusted for the different sets of simulation results. In addition, we selected a value of $\langle f\rangle=1 / 3$ to represent the effective fraction of collectors oriented in the same direction as that used in the single-collector analysis (perpendicular both to the flow direction and parallel to the acoustic pressure nodes). All other parameters in the model are set to values obtained through measurements of system parameters (e.g. $v_{0}, \varepsilon_{0}, C_{0}$, and $R_{c}$ ), independent experiments (e.g. $N_{T}$ ), or calculated from independent theory(e.g. $R_{\text {cap }}^{*}$ ).

\subsection{Data from Gupta and Feke (1998)}

Table 1 summarizes the experimental conditions used in this study. These experiments were performed according to the second scenario described above, namelythe suspension flows through the mesh prior to energizing the acoustic field.

The single-collector model (Grossner et al., 2003) was used to determine the dimensions of the capture window for the particular acoustic and flow environment. The energydensityinput for this calculation was found using a multi-layer resonance model developed by Rusinko (2001). Subsequently, the algorithm described above was applied to
Table 1

Parameters used in the experiments by Gupta and Feke (1998)

\begin{tabular}{lc}
\hline $\begin{array}{l}\text { Feed suspension } \\
\text { Flow rate }\end{array}$ & $\begin{array}{l}0.5 \mathrm{wt} \% 325 \text {-mesh polystyrene } \\
30 \mathrm{~mL} / \mathrm{min}\end{array}$ \\
& \\
$\begin{array}{l}\text { Chamber dimensions } \\
\text { Mesh composition }\end{array}$ & $5.82 \mathrm{~mm} \times 35 \mathrm{~mm} \times 70 \mathrm{~mm}$ \\
& Polyester, 10 pores per inch \\
Power supplied to transducer & $20 \mathrm{~W}$ \\
Frequency 1.103 & $\mathrm{MHz}$
\end{tabular}

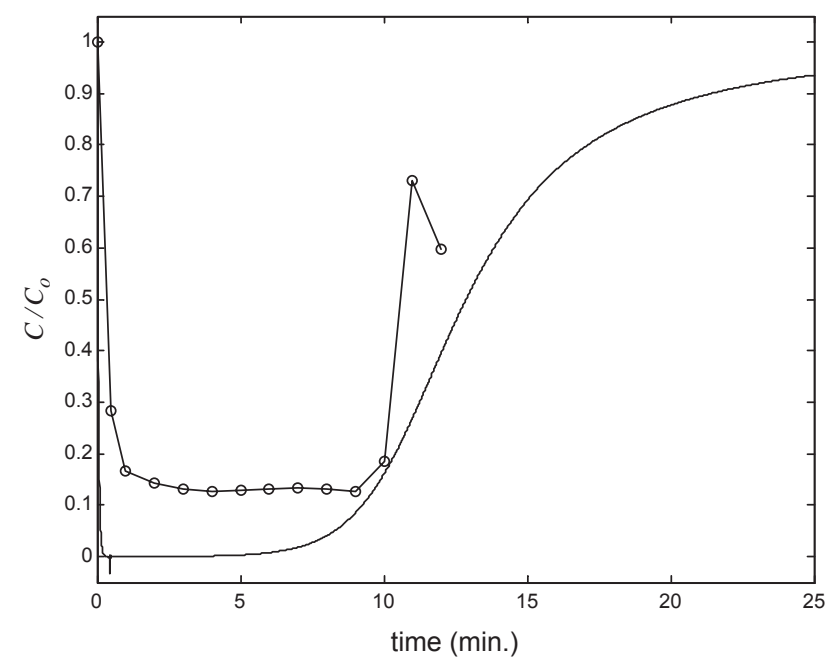

Fig. 3. The normalized concentration at the separator exit for a flow rate of $30 \mathrm{~mL} / \mathrm{min}$ and an inlet concentration of $0.5 \mathrm{wt} \%$. Other conditions of the experiment are listed in Table 1 . The circles are experimental data (Gupta and Feke, 1998) and the unmarked line is the output of the present model. Without anyparameter adjustments, breakthrough time is predicted quite well, but the actual concentration does not drop as significantlyas the simulation predicts.

obtain profiles of $C(x, t)$ and $N(x, t)$. Based on experimental results, the value of $N_{T}$ was taken to be $15 \mathrm{vol} \%$ for the particular mesh used in these experiment. Results are presented in dimensionless form, using $C_{0}$ and $N_{T}$ as normalizing scales.

Fig. 3 presents the predicted concentration of particles in the exit stream from the mesh $\left(C(x=L, t) / C_{0}\right)$ for the conditions listed in Table 1 along with the corresponding experimental data. Figs. 4 and 5 show predicted and experimental results for the case when the flow rate increased to $60 \mathrm{~mL} / \mathrm{min}$ and the feed concentration increased to $1.0 \mathrm{wt} \%$, respectively.

In Fig. 3, the normalized concentration, $C / C_{0}$, begins at 1 and is predicted to sharplydecline toward zero for the first $20 \mathrm{~s}$. A discontinuityin the output is seen at $0.5 \mathrm{~min}$, which corresponds to the residence time of the suspension in the chamber. After 5-6 min with no particles exiting the separator, a gradual rise in the concentration appears before a sharp increase begins near $10 \mathrm{~min}$ of operation. The normal- 


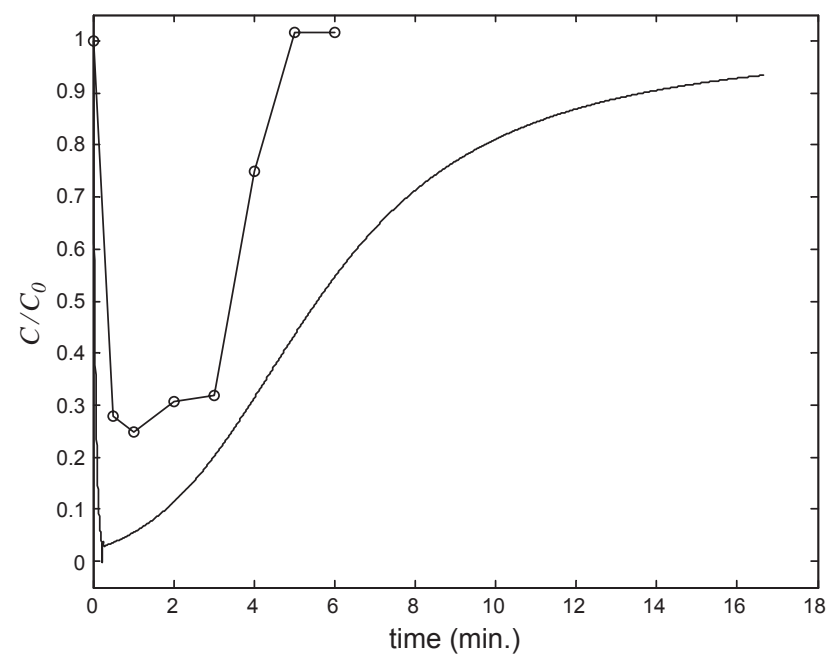

Fig. 4. The normalized concentration at the separator exit for a flow rate of $60 \mathrm{~mL} / \mathrm{min}$ and an inlet concentration of $0.5 \mathrm{wt} \%$. Other conditions of the experiment are listed in Table 1. In this case, the experimental output concentrations (circles) are again slightlyhigher than expected from model predictions (unmarked line).

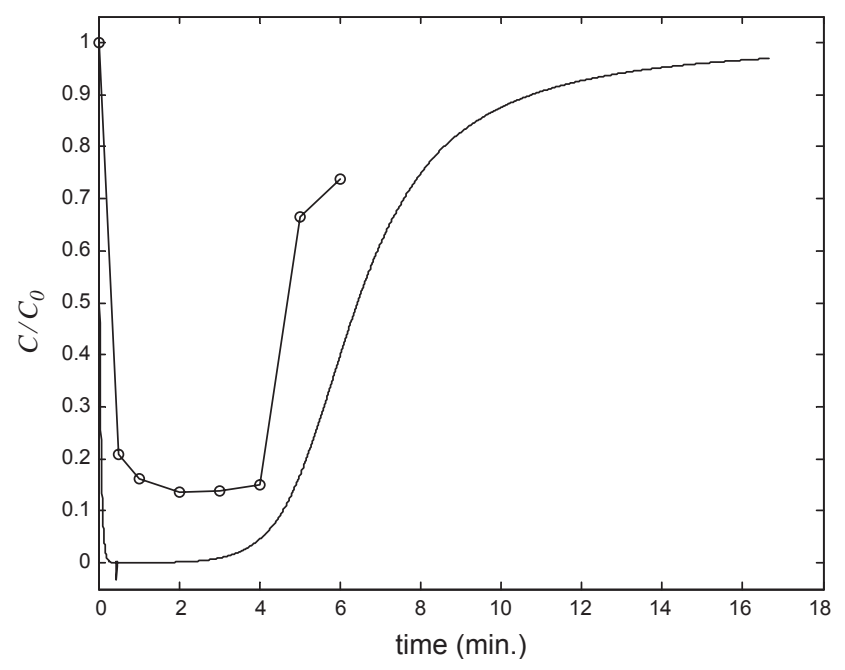

Fig. 5. The normalized concentration at the separator exit for a flow rate of $30 \mathrm{~mL} / \mathrm{min}$ and an inlet concentration of $1.0 \mathrm{wt} \%$. Other conditions of the experiment are listed in Table 1. Again, the model predicts a lower concentration (unmarked line) than the experiment (circles), but the breakthrough time is appropriatelyscaled relative to that in Fig. 3.

ized concentration asymptotically approaches 1 as the time continues past $20 \mathrm{~min}$.

One quantitative difference between experimental results and model predictions is that, after a rapid initial drop in exit concentration from $C_{0}$, the model shows $100 \%$ efficiencyin particle retention until saturation and breakthrough begins to occur. The experimental data show approximately $85 \%$ retention efficiencyduring the corresponding period. However, the main features of the experimental results, a sharp decline in exit concentration, followed bya plateau and then a breakthrough period, are predicted bythe model. In his ex-
Table 2

Breakthrough time comparison between simulation and experiment

\begin{tabular}{|c|c|c|c|}
\hline \multirow{2}{*}{$\begin{array}{l}\text { Flow rate } \\
(\mathrm{mL} / \mathrm{min})\end{array}$} & \multirow{2}{*}{$\begin{array}{l}\text { Feed concentration } \\
(\mathrm{wt} \%)\end{array}$} & \multicolumn{2}{|c|}{ Breakthrough time } \\
\hline & & $\begin{array}{l}\text { Experiment } \\
(\mathrm{min})\end{array}$ & $\begin{array}{l}\text { Model } \\
(\min )\end{array}$ \\
\hline 30 & 0.5 & 10 & 9 \\
\hline 60 & 0.5 & 3 & 1.5 \\
\hline 30 & 1.0 & 4 & 4.5 \\
\hline
\end{tabular}

periments, Gupta recorded the exit concentration as a function of time up to, but not beyond, the breakthrough point.

The time to breakthrough can be used to gauge the correspondence between model and experiment. Particularly, changes in experimental conditions give changes in breakthrough times, so if the simulation predicts the same changes, this is an indication of the applicabilityof the model. Table 2 lists breakthrough times from experiments and model calculations for different flow rates and feed concentrations.

The breakthrough times compare well between model and experiment; it is important to note that no model parameters were adjusted to fit the data between experimental results. As one might expect, doubling the feed concentration halves the time to breakthrough in both the experiment (approximately) and in the model. Since twice as manyparticles pass through the chamber in the same amount of time, the capacityof the filter is reached in half the time.

Envisioning the effect of doubling the flow rate, however, is not as straightforward. One might expect again that the time to fill the capacityof the separator would be halved. According to both the experiment and the model, however, this is not the case. In Gupta's work, the breakthrough time is reduced to $30 \%$ of the original value when the flow rate is doubled, whereas the model predicts breakthrough time should be reduced to nearly $20 \%$ for the corresponding increase in flow rate. This can be interpreted bye xamining what is happening on the microscale in the single-collector model. The dimensionless capture radius was calculated using the single-collector model (Grossner et al., 2003) to be 2.8 for a $30 \mathrm{~mL} / \mathrm{min}$ flow rate, but only 0.98 when the flow was doubled to $60 \mathrm{~mL} / \mathrm{min}$. So, not onlyis the separator exposed to more particles per unit time, the effectiveness of each collector element is reduced bythe increase in linear flow speed. Both of these factors affect the breakthrough time, and the model predicts this behavior quite appropriately.

\subsection{Data from Grabenstetter (2004)}

Table 3 summarizes the conditions used for these experiments. Here, the suspension was fed through the mesh prior to the application of the acoustic field, so the second set of boundaryconditions described above applies to this case as 
Table 3

Parameters used in the experiment by Grabenstetter (2004)

\begin{tabular}{ll}
\hline Feed suspension & $0.21 \mathrm{wt} \% 74 \mu \mathrm{m}$-diameter polystyrene \\
Flow rate & $35 \mathrm{~mL} / \mathrm{min}$ \\
& \\
Chamber dimensions & $25.5 \mathrm{~mm} \times 22.3 \mathrm{~mm} \times 42.2 \mathrm{~mm}$ \\
Mesh composition & Polyester, 10 pores per inch \\
& \\
Energydensity & $0.02 \mathrm{~J} / \mathrm{m}^{3}$ \\
Frequency 1.100 & $\mathrm{MHz}$ \\
\hline
\end{tabular}

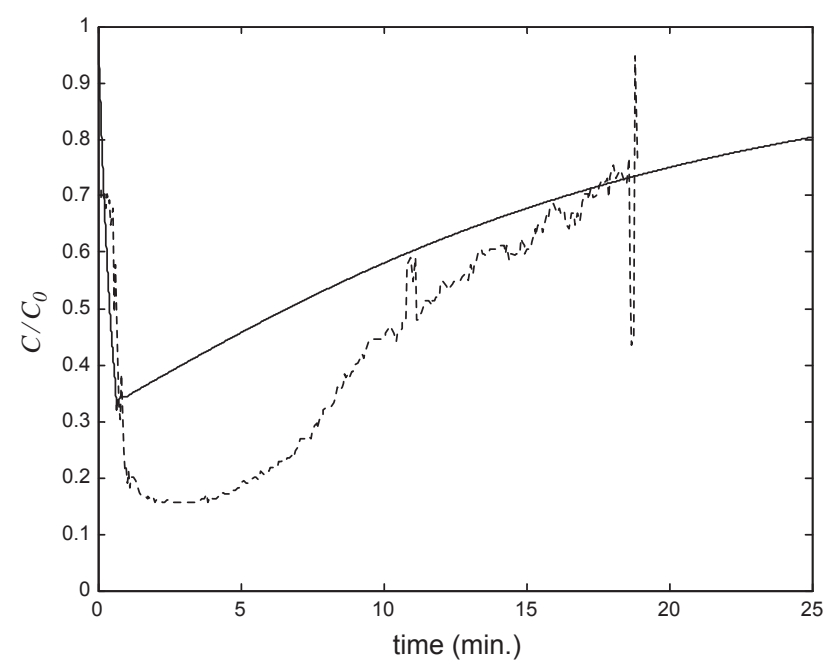

Fig. 6. The normalized concentration at the exit of the separator is shown for both the integrated model (solid line) and the experiment (dashed line) with conditions shown in Table 3 (Grabenstetter, 2004). Here the model predictions are performed using $N_{T}=0.05$.

well. The acoustic energydensityin the fluid was estimated using the model of Rusinko (2001). The saturation limit of this particular mesh was not explicitlydetermined, but experimental observations indicate that it is lower than that of the previous set of examples. For the purposes of illustration, we performed simulations using a value of $N_{T}=0.05$.

Model predictions are shown in Fig. 6. As before, the model predicts a sharp drop off in exit particle concentration followed bya protracted period in which retention efficiencyis relativelyhigh. In this case, the normalized exit concentration $C / C_{0}$ is predicted to drop to 0.32 , which is similar to the experimental result of 0.20 . Also, both the model and experiments show a nearlylinear increase with time following this initial drop. Since these experiments were performed in a larger chamber, the acoustic energy densityis somewhat smaller than that used in the set of experiments reported by Gupta and Feke (1998). This results in a less effective capture, and consequently, only the trailing edge of the S-shaped curve seen in Figs. 3-5 is evident. Here too, the correspondence between the experimental and simulation results is quite good.

\section{Summary and conclusions}

Using results from a single-collector trajectoryanaly sis, a macroscopic transport model was derived in order to predict the performance of the ultrasonicallyenhanced mesh filtration process. This overall model was used to predict the exit concentration versus time for experiments previously reported in the literature. The model predicts well the general features of the experimental data. The time to breakthrough predicted bythe model was verynear to that seen in the experiments, and also scaled properlywith respect to changes in flow rate and feed concentration. Slight discrepancies between the model predictions and experimental results can be attributed to imperfections in the experimental apparatus. However, adjustment of model parameters could be performed in order to yield a better correspondence of predictions to experimental data.

\section{Acknowledgements}

This work was supported byN ASA through Grant NAG $8-1584$.

\section{References}

Gerber, R., Birss, R.R., 1983. High Gradient Magnetic Separation. Wiley, New York.

Gerber, R., Watmough, M.H., 1982. Magnetic separator equations. IEEE Transactions on Magnetics 18 (6), 1671-1673.

Grabenstetter, P.J., 2004. Unpublished Data.

Grossner, M.T., Penrod, A.E., Belovich, J.M., Feke, D.L., 2003. Single fiber model of particle retention in an acousticallydri ven porous mesh. Ultrasonics $41,65-74$.

Grossner, M.T., Feke, D.L., Belovich, J.M., 2005. Single-collector experiments and modeling of an ultrasonicallyenhanced filtration process. A.I.Ch.E. Journal, in press.

Gupta, S., Feke, D.L., 1997. Acousticallydri ven collection of suspended particles within porous media. Ultrasonics 35, 131-139.

Gupta, S., Feke, D.L., 1998. Filtration of particulate suspensions in acousticallydri ven porous media. A.I.Ch.E. Journal 44, 1005-1014.

Rusinko, D., 2001. Design and optimization of an ultrasonic standing wave chamber. M.S. Thesis, Case Western Reserve University, Cleveland, $\mathrm{OH}$, USA.

Wang, Z., Grabenstetter, P.J., Feke, D.L., Belovich, J.M., 2004. Retention and viabilitycharacteristics of mammalian cells in an acoustically driven polymer mesh. Biotechnology Progress 20, 384-387. 